\title{
Analysis of the Post-Critical Deformation State of Curvilinear Integral Stiffening of a Thin-Walled Structure Subjected to Torsion
}

\author{
Tomasz Kopecki ${ }^{1}$
}

1 Department of Aviation and Mechanical Engineering, Rzeszów University of Technology, al. Powstańców Warszawy 12, 35-959 Rzeszów, Poland

e-mail: tkopecki@prz.edu.pl

\begin{abstract}
The paper presents an innovative concept of integral stiffening of the thin-walled structures based on the use of curvilinear elements. The analyzed system in the form of a thin-walled spatial structure was subjected to a nonlinear numerical analysis, aimed at comparing the properties of the proposed solution with those of the reference stiffening. A comparative analysis of deformation forms and ranges as well as effective stress distributions was conducted. The tests constituted a preliminary analysis, aimed at determining the usability of the solution, which is the basis of the research process supported by an appropriate experiment.
\end{abstract}

Keywords: isogrid, advanced deformation, finie elements method, nonlinear analysis

\section{INTRODUCTION}

Many types of the currently designed thinwalled load-bearing structures use an increasingly popular method of increasing their rigidity, which is using integral rod elements forming a kind of grid. This type of solution called "isogrid" is used in particular in the structures subjected to large deformations caused by the loss of stability. Integral stiffeners allow to significantly increasing the value of critical loads at a relatively low increase of the structure mass. This feature caused the isogrid to become a commonly used solution in the load-bearing structures for which the criterion of low mass is of fundamental importance, i.e. in aviation constructions.

The problem of the loss of stability of the thin-walled working covering appeared with the beginning of the use of aeronautical semi-monocoque and monocoque structures. The attempts to completely eliminate the phenomenon of buckling led to a significant increase in the weight of the structure; therefore, various ideas for stiffening appeared, among which the initially most common solution was using a fluted sheet metal (e.g. in Junkers Ju-52 and Ford Trimotor aircrafts).
The increase in the knowledge of design methods and the nature of certain physical phenomena over time has allowed for the adoption of the construction doctrine, which is still used today in the case of metal structures, according to which the phenomenon of loss of covering stability is permissible if it has an elastic character and occurs locally within a segment limited with skeletal elements such as frames and stringers $[1,10,12]$ (Fig.1).

Acceptance of the admissibility of this phenomenon entails the risk of permanent deformations resulting from the fatigue effects. Therefore, it has become strongly advisable to reduce the amount of critical deformations by seeking the solutions that increase the rigidity of the covering without a growth, or with a minimal increase in its mass. The simplest of them turned out to be the use of the above-mentioned fluted sheet; however, this variant of stiffening caused a significant decrease of the aerodynamic properties of the aircraft. Another way to increase the rigidity of airframe coatings was to perform deep pressing $[4,5,6]$.

The development of manufacturing techniques, and in particular the emergence of precise, 


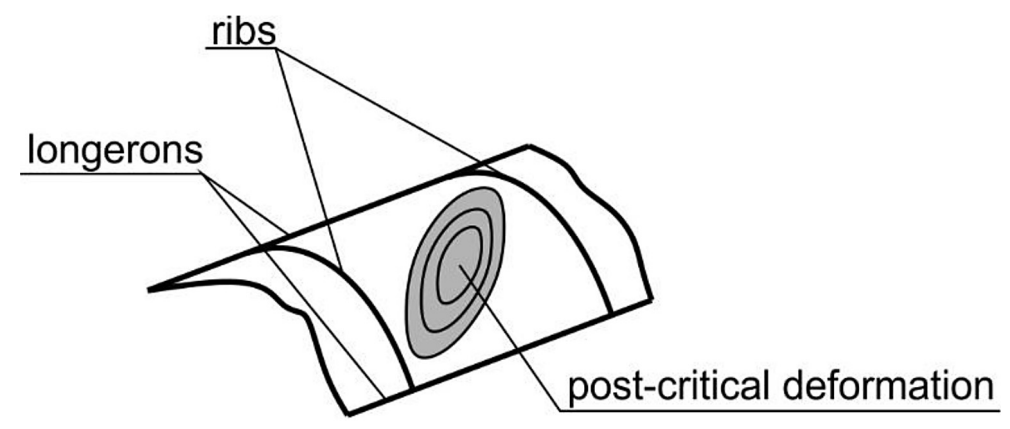

Fig. 1. Acceptable, local form of loss of coverage stability

numerically controlled machine tools, created the possibility of free shaping of cover elements, which resulted in an increased use of integral isogrid stiffeners (Fig.2).

Integral stiffeners have also found application in the coverings made of various types of composites. In the case of aviation load-bearing structures, this solution is an alternative to the sandwich structures containing additional layers of polymer foams or other materials preventing the loss of covering stability [13,17].

A comprehensive research on the properties of this solution was initially conducted by NASA. Over time, the issue of integral stiffeners began to become popular also in university centers, which resulted in its appearance of many scientific publications $[9,15,18]$.

When analyzing the geometric forms of the utilised types of isogrid stiffeners, it should be noted that the vast majority of them are based on the use of rectilinear beam elements. Depending on the variant of the solution used and the type of the load, the critical elements of the stiffening grid are either its elements or the nodes connecting them. In general, the higher the density of the integral stiffening grid, the lower the likelihood of unwanted deformations; however, increasing its density is directly related to the increase in the mass of the structure. Assuming that the mass of stiffeners can only constitute a certain percentage of the mass of the coverage fragment, it seems particularly important to adopt the most effective geometric form of the grid.

In this study, an unusual, innovative solution involving the use of stiffening elements in the form of curvilinear rods creating a repeatable pattern was proposed. Although the scientific publications include the studies on curvilinear isogrid [], the solutions analyzed there are fundamentally different from those proposed in this study. Two variants of the structure were subjected to the non-linear numerical analyses: the reference one, based on a typical solution, containing rectilinear elements, and the examined one, using a grid of curvilinear elements.

The results of the analysis showed that in the case of the proposed new solution, the obtained form of critical deformation results in a more favorable distribution and lower values of stress. The presented results constitute a preliminary analysis aimed at determining the advisability of conducting broader research on similar structures, in particular conducting the experimental research of a specific spectrum of variants of the proposed solution. The applied geometric model was adopted with the available research tools in mind.

\section{Purpose and scope of the study}

The subject of comparative analysis were two numerical models of thin-walled system reinforced with isogrid stiffening (Fig. 3-4).
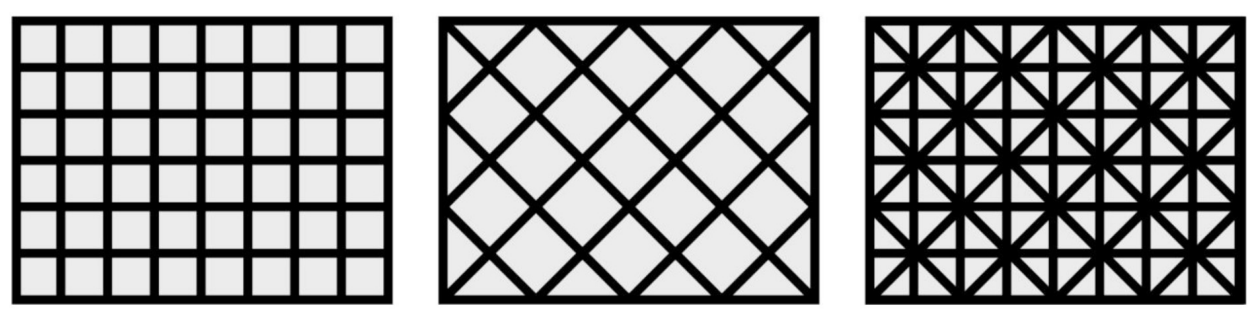

Fig. 2. Examples of typical variants of isogrid stiffeners 
The first version of the model, treated as the reference, is based on a typical solution with rectilineal elements of the stiffening grid. In the second version, an isogrid made of curvilinear elements was used. Both models were in form of cuboid with side of $184 \mathrm{~mm}$ length. Because performing the experiments with use of printed models is planned in the next stage of the research, the dimensions of the model walls corresponding to a size of a working space of a 3D printer were selected.

The front and rear walls served as ribs and had a constant thickness of $2 \mathrm{~mm}$. The side walls were $0.4 \mathrm{~mm}$ thick, while the cross-sectional dimension of both isogrid variants, corresponding to the normal direction to the wall plane, was $1 \mathrm{~mm}$. In turn, the dimension parallel to the plane of the cross-section wall of the stiffening elements was:
$1.2 \mathrm{~mm}$ for the reference structure and $0.6 \mathrm{~mm}$ for the tested structure (Fig. 4, 5).

The model stiffened by curvilinear isogrid had twice as many grid elements as the reference model, with twice smaller the cross-sectional area and therefore, both models had the same mass. In both cases, an identical mounting and load scheme was adopted (Fig. 6). The models were subjected to torsion by applying a pair of forces of $200 \mathrm{~N}$. The fixing was carried out by eliminating the translational degrees of freedom on the directions of the three axes of the reference system of the selected nodes within the rear wall of the model. In addition, displacements in the plane of the frontal frame of its central node were blocked.

Although the planned experiments assume use of typical 3D printing materials, the initial numerical analysis used the physical constants corresponding to polycarbonate, which is

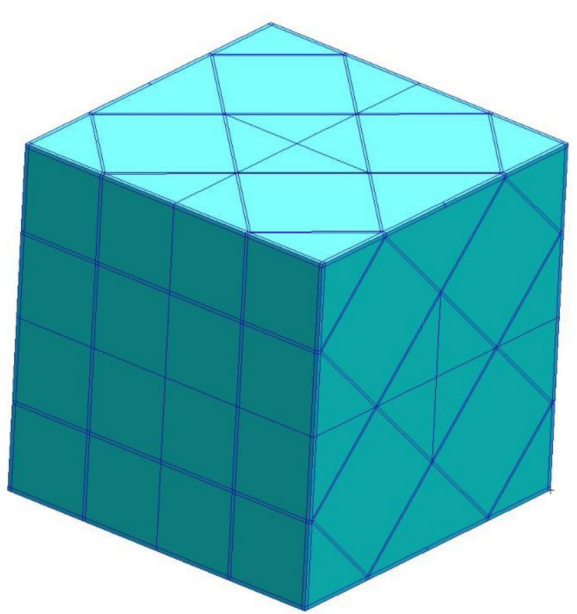

a)

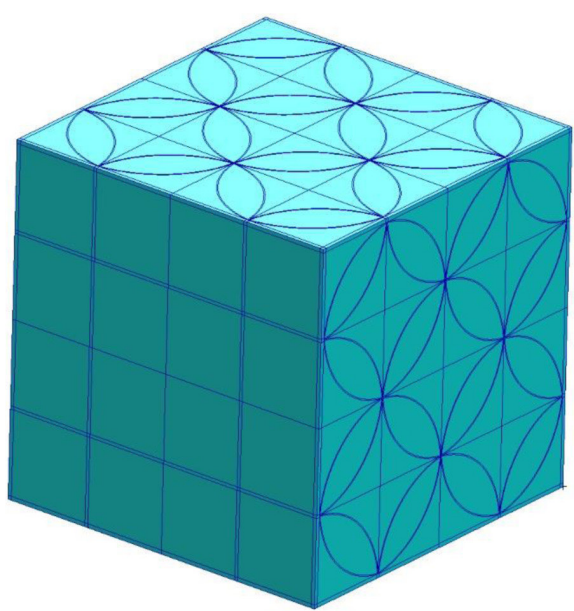

b)

Fig. 3. Numerical models of the analyzed systems: a) reference model, b) model with curvilinear isogrid

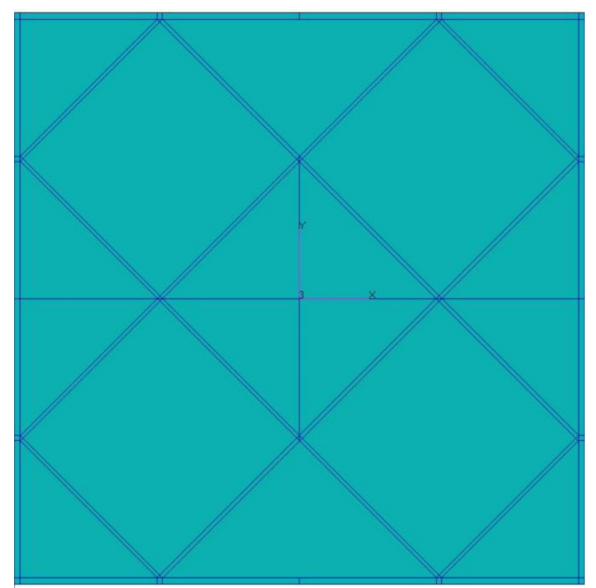

a)

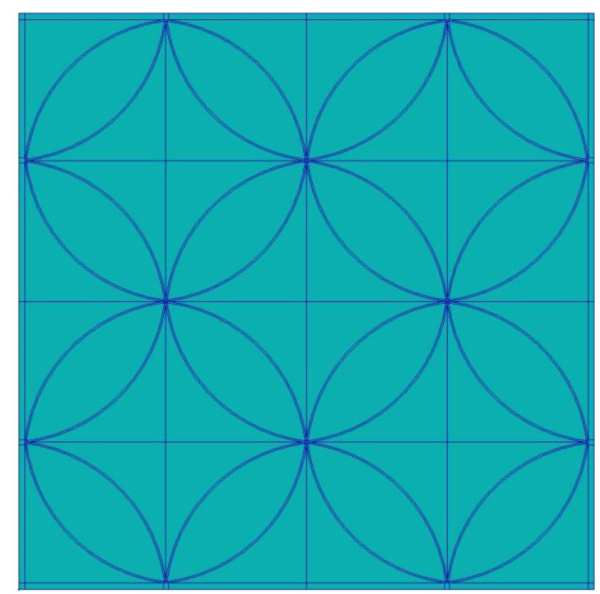

b)

Fig. 4. Geometric models of the side walls of the analyzed system: a) reference model, b) model with curvilinear grid 


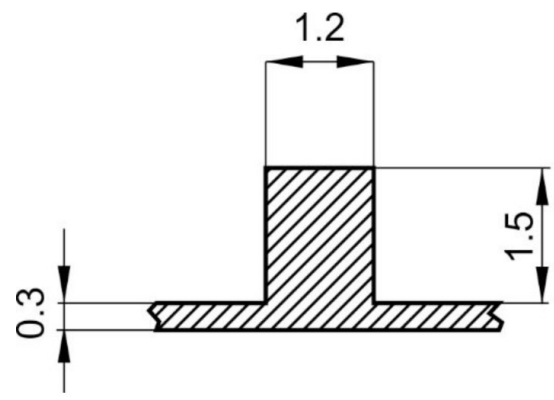

a)

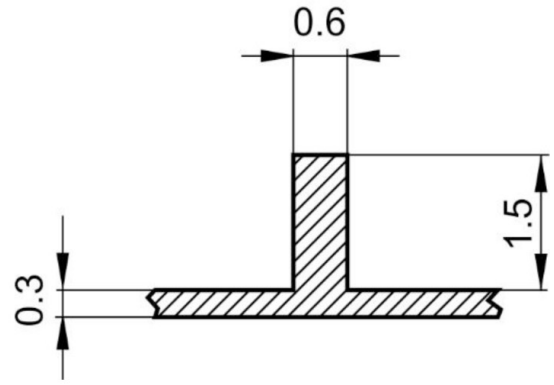

b)

Fig. 5. Cross-sections of wall fragments in the area of the stiffening: a) reference model, b) model with curvilinear grid

a commonly used model material. Therefore, a linear-elastic model was adopted, described by constants: $\mathrm{E}=2150 \mathrm{MPa}, \mathrm{v}=0.38$.

Under the adopted conditions, the side walls are sheared due to the twisting of the system, which leads to a loss of stability due to the appearance of a draw field in the diagonal direction of the wall. Therefore, the static properties of curvilinear beams, which are significantly influenced by the size of the radii of curvature of the grid elements, can have a decisive impact on the distribution of stress [16].

The considered geometric form of the examined variant of integral stiffening results from the preliminary assumptions and its modification is assumed in the course of further research in order to determine the most favourable solution from the point of view of both the form of deformation and stress distribution.

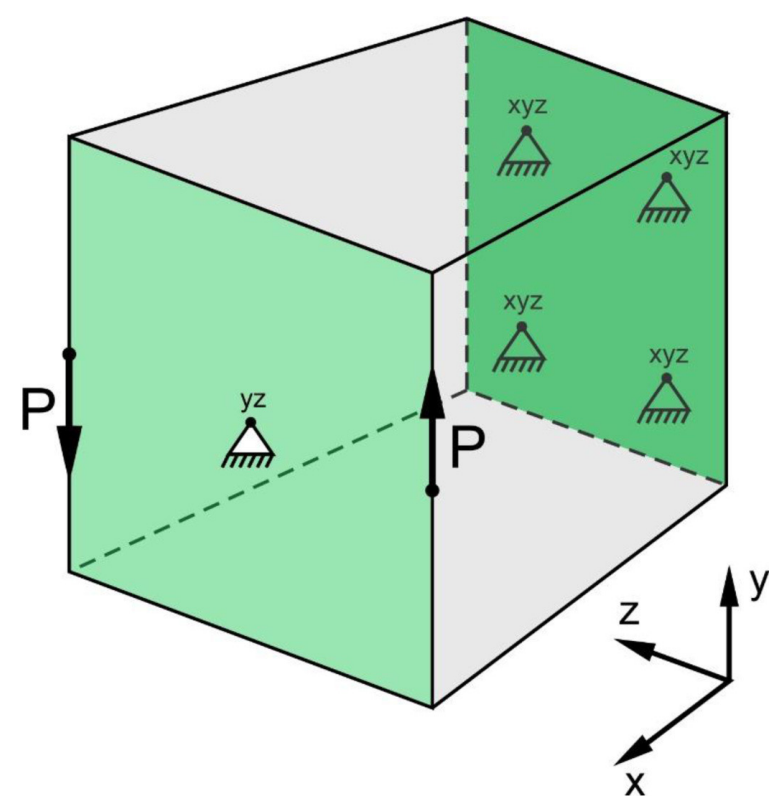

Fig. 6. Diagram of load and application of the constraints

\section{Numerical calculations}

The considered problem was characterized by a non-linearity of a geometric nature, resulting from the large deformations caused by the loss of stability of the studied systems. All calculations were performed using the MSC PATRAN/MARC software, based on the finite elements method [2].

From a numerical point of view, the essence of solving a non-linear problem is to determine the instantaneous static equilibrium of the system resulting from the relationship between a set of parameters determining the state of the structure (the state parameters) and a set of load related parameters (the control parameters). Therefore, the static equilibrium of the system occurs when the matrix equation of the residual forces is met in the following form:

$$
r(u, \Lambda)=0
$$

In this equation $r$ is a residual vector, i.e. a vector with components corresponding to the unbalanced components of forces at the current state of deformation of the system, while $u$ and $\Lambda$ are: vector of state parameters and matrix of control parameters related to the load, respectively $[3,7,8,11]$.

Equation (1) can take the form of monoparameter residual force equation in which the control parameter matrix is replaced by a single parameter $\lambda$. on which all load components affecting the structure depend:

$$
r(u, \lambda)=0
$$

The application of the iterative correction phase corresponds to the fulfillment of an additional equation, i.e. increment control equation, also called constraints equation, specifying the conditions adopted by the user for determining the instantaneous equilibrium. If the incremental values of the state parameters and the control 
parameter corresponding to the transition from the state $\mathrm{n}$ to state $\mathrm{n}+1$ are defined as:

$\Delta u_{n}=u_{n+1}-u_{n}$ and $\Delta \lambda_{n}=\lambda_{n+1}-\lambda_{n}$

the equation of constraints can be written as:

$$
c\left(\Delta u_{n}, \Delta \lambda_{\mathrm{n}}\right)=0
$$

Equation (3) is a hypersurfacing equation in the hyperspace of the state, corresponding to successive equilibrium states determined as load increases, called the system's equilibrium path. It can be saved in a changed form:

$$
\mathbf{a}^{\mathrm{T}} \cdot \stackrel{\circ}{\mathbf{u}}+\mathrm{g} \cdot \stackrel{\circ}{\lambda}=0
$$

where:

$$
\mathbf{a}^{\mathrm{T}}=\frac{\partial \mathrm{c}}{\partial \mathbf{u}} \quad ; \mathrm{g}=\frac{\partial \mathrm{c}}{\partial \lambda}
$$

In the case of the discussed analyses, the Newton-Raphson prediction method with an added corrective strategy based on state control was used.

In both the reference and the tested structure, the finite element grid consisted of bilinear thinwalled shell elements with six degrees of freedom in each of the nodes. In both cases, a grid consisting mostly of quadrangular elements was used. The presence of triangular elements was allowed in the areas where specific geometric form prevented the division into quadrangles. The number of nodes for both models was similar and amounted to: 51488 - for the reference structure and 60603 - for the model with curvilinear isogrid (Fig. 7). As a result of the non-linear numerical analyses, the displacement distributions were obtained (Fig. 8). In order to subject the results obtained for both solutions to a detailed comparative analysis, the respective walls of the systems were isolated (Fig. 9).

In order to clearly show the differences between the results of analyses, all the statements were prepared using the same colour scale ranges for the both analyzed systems. Attention should be paid to the significant differences in the distributions presented above. In both cases, the general form of critical deformation was manifested by the formation of three folds with the orientation corresponding to the direction of the drawing field. The main difference was in the proportions between the extent of the folds and their depths.

In the case of the structure under study, the maximum displacement in the area of the central fold was only about $8 \%$ larger than of the reference structure. At the same time, the deformation area was clearly larger, resulting in lesser curvature and therefore lower stresses due to the flexural effect. In the lower fold area (according to Figure 9), the maximum displacement in the reference structure was nearly $70 \%$ higher than in the system with curvilinear isogrid. The described differences seem to result mainly from the static properties of the beams constituting the integral stiffener. The advantages of the proposed solution are also confirmed by the distribution of reduced stress, according to the $\mathrm{Hu}-$ ber-Mises-Hencky hypothesis (Fig. 10).

The numerical analysis showed that the maximum stress values for a structure with a curvilinear isogrid are only approx. $40 \%$ in relation to the reference structure. Additionally, the much more favorable nature of the distribution is noteworthy, lower levels of stress in the fold area on the diagonal wall and the lack of their concentration in the corners. In the central part of the wall, in the

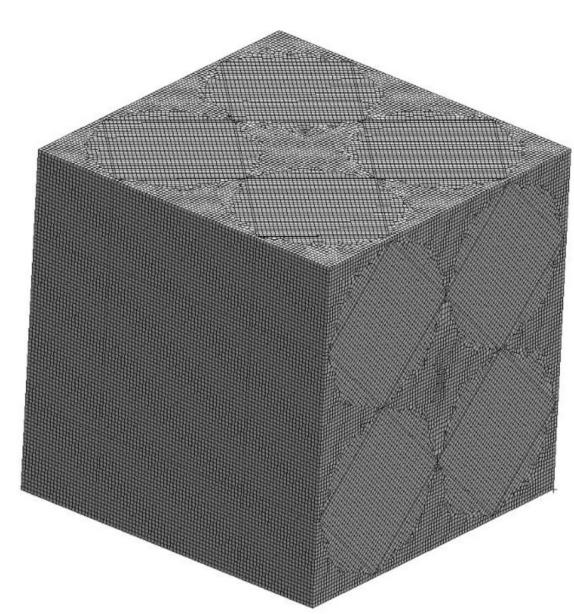

a)

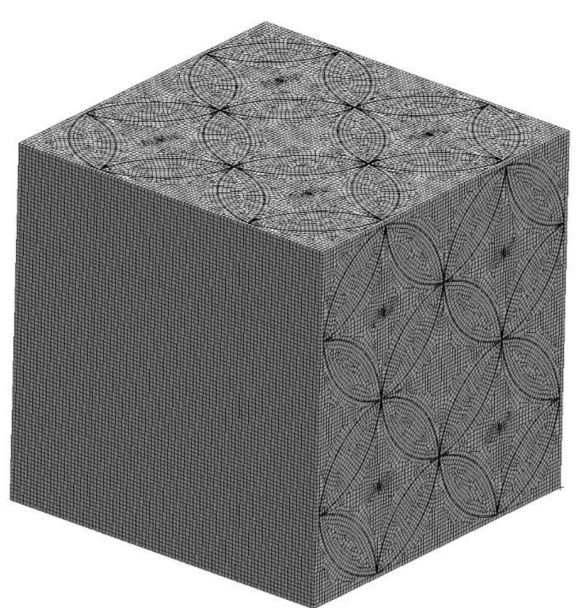

b)

Fig. 7. Finite element grids: a) reference model, b) model with curvilinear isogrid 


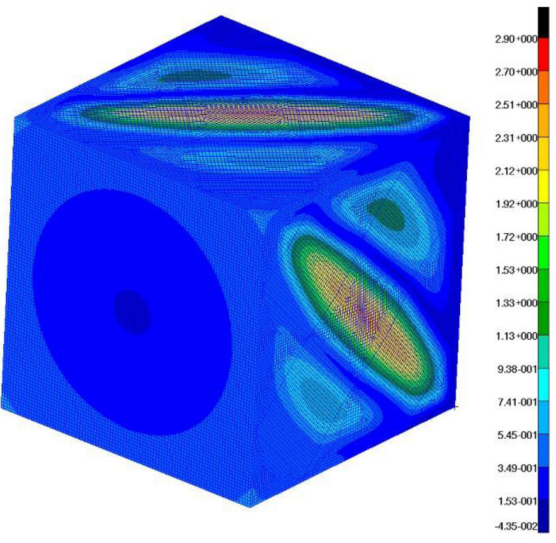

a)

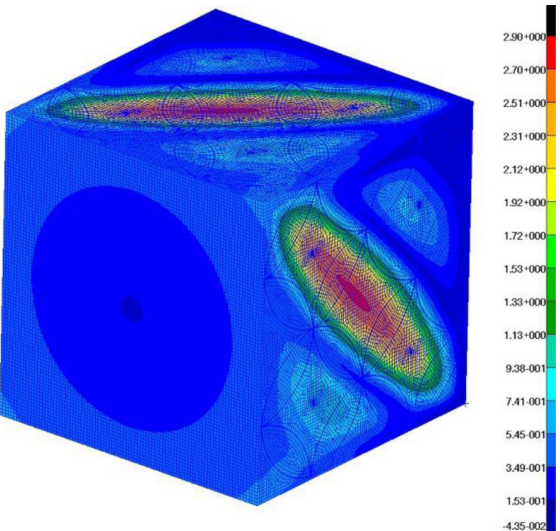

b)

Fig. 8. Distributions of total displacements: a) reference model, b) model with curvilinear isogrid

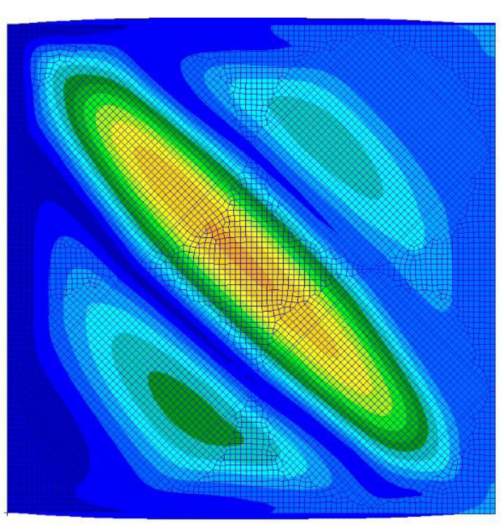

a)
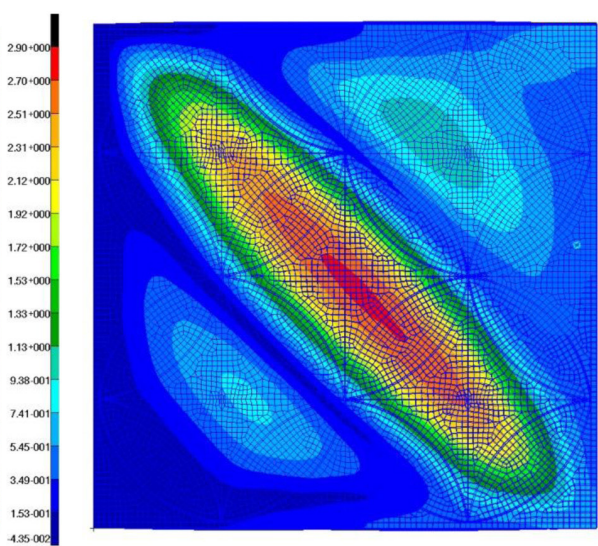

b)

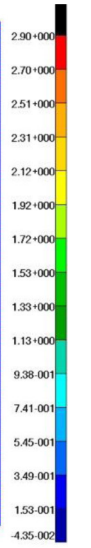

Fig. 9. Distributions of total displacements in the side wall areas of the analyzed systems a) reference model, b) model with curvilinear isogrid area of the covering without stiffening, the largest value of reduced stress is approx. $70 \%$ of the value for the reference structure, and the nature of the distribution is significantly more favourable, without a clear gradient.

\section{CONCLUSIONS}

The presented results of the non-linear numerical analyses prove that the proposed variant of stiffening the structure with curvilinear isogrid has significant advantages compared to the traditional solutions. It should be emphasized that the segments of airframe coverings are subjected to repeated critical deformation cycles under operating conditions. Therefore, there is a concern that in the case of too large stress gradients in these systems, the fatigue damage may occur [14]. The search for new solutions should therefore be based on the criterion of seeking to reduce the maximum values and stress gradients. An effective way to achieve this goal seems to be adopting the appropriate variant of integral stiffening. The presented solution allows for a clear improvement in the nature of stress distribution without the need to increase the mass of the structure.

The described problem concerns not only the structures made of isotropic materials, but also the composites in which damage resulting from cyclic loads may mean delamination of the structure and the associated rapid reduction of its loadbearing capacity, leading to destruction.

The proposed variant of integral stiffening is one of many possible solutions. Because the static properties of its constituent elements have a decisive influence on the effectiveness of the stiffening grid, the factors of deformation and stress distribution are their radius of curvature. Therefore, it seems advisable to carry out further numerical analyses of subsequent solution variants, aiming at determining the impact of the geometric 


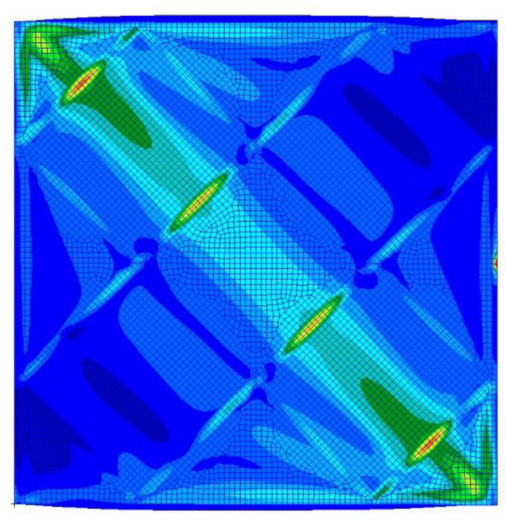

a)

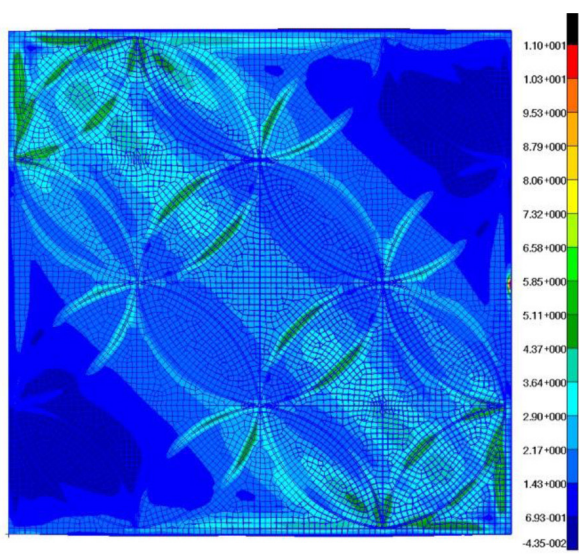

b)

Fig. 10. Reduced stress distributions according to the Huber-Mises-Hencky hypothesis in the side walls of the analyzed systems a) reference model, b) model with curvilinear isogrid

parameters of the curvilinear isogrid on the mechanical properties of the examined system. As a result, it will be possible to obtain the most favourable solution from the point of view of the said criterion. It also seems necessary to conduct an experiment that is a factor verifying the correctness of the analysis of the final solution.

\section{REFERENCES}

1. Arborcz J. Post-buckling behavior of structures. Numerical techniques for more complicated structures. Lecture Notes in Physics, 288, 1985, 83-142.

2. Crisfield M.A. Non-linear finite element analysis of solid and structures. J. Wiley \& Sons, 1997.

3. Doyle J.F. Nonlinear analysis of thin-walled structures. Springer-Verlag, 2001.

4. Dębski H., Sadowski T. Modelling of microcracks initiation and evolution along interfaces of the $\mathrm{WC} /$ Co composite by the finite element method. Computational Materials Science, 83, 2014, 403-411.

5. Dębski H., Teter A., Kubiak T. Numerical and experimental studies of compressed composite columns. Composite Structures, 118, 2014, 28-36.

6. Dobrzański P., Czarnocki P., Lorenz Z. Shell structures - theory and application. CRC Press, 2013.

7. Felippa C.A. Procedures for computer analysis of large nonlinear structural system in large engineering systems. A. Wexler (Ed.), Pergamon Press, London, UK, 1976.

8. Felippa C.A., Crivelli L.A., Haugen B. A survey of the core-congruential formulation for nonlinear finite element. Archive of Computer Methods in Engineering, 1994.

9. Huybrechts S., Tsai S.W. Analysis and behavior of grid structures. Composites Science and Technology, 56, 1996, 1001-1015.
10. Lynch C.A. Finite element study of the post buckling behavior of a typical aircraft fuselage panel. PhD Thesis, Queen's University Belfast, 2000.

11. Kopecki T. Numerical-experimental analysis of the post-buckling state of a multi-segment multi-member thin-walled structure subjected to torsion. Journal of Theoretical and Applied Mechanics; 49(1), 2011, 227-242.

12. Kopecki T., Bakunowicz J., Lis T. Post-critical deformation states of composite thin-walled aircraft load-bearing structures. Journal of Theoretical and Applied Mechacnics, 54(1), 2016, 195-204.

13. Kubit A., Trzepiecinski T., Święch Ł., Faes K., Slota J. Experimental and numerical investigations of thin-walled stringer-stiffened panels welded with RFSSW technology under uniaxial compression. Materials, 12(1785), 2019.

14. Mazurek P. Fatigue strength of thin-walled rectangular elements in the state of post-critical deformation. Advances in Science and Technology-Research Journal, 13(2), 2019, 84-91.

15. Singh K., Kapania R.K. Optimal design of towsteered composite laminates with curvilinear stiffeners. AIAA/ASCE/AHS/ASC Structures, Structural Dynamics, and Materials Conference, 8-12 January 2018.

16. Święch $Ł$. Experimental and numerical studies of low-profile, triangular grid-stiffened plates subjected to shear load in the post-critical states of deformation. Materials, 12(3699), 2019.

17. Teter A., Debski H., Samborski S. On buckling collapse and failure analysis of thin-walled composite lipped-channel columns subjected to uniaxial compression. Thinn-Walled Structures, 85, 2014, 324-333.

18. Wang D., Abdalla M., Zhang W., Buckling optimization design of curved stiffeners for gridstiffened composite structures. Composite Structures, 2016. 